\title{
Event-based Distributed Filtering over Markovian Switching Topologies
}

\author{
Qinyuan Liu, Zidong Wang, Xiao He and D. H. Zhou
}

\begin{abstract}
In this paper, we consider the distributed filtering problem for continuous-time stochastic systems over sensor networks subject to Markovian switching topologies. Due to limited communication energy and bandwidth, an event-based communication scheme is proposed with the aim to decrease the transmission frequency. An individual triggering condition is put forward to regulate the communication rates for each component of the system state in order to better reflect the engineering requirements. The aim of this paper is to design a distributed filter over sensor networks with Markovian switching topologies such that the dynamics of the estimation error is exponentially mean-square bounded. It is shown that, with the proposed event-based distributed filtering algorithm, the exponential meansquare boundedness of the estimation errors is guaranteed if the sensor network is distributively detectable and the combined communication topology is strongly connected. A numerical example is presented to illustrate the usefulness of the developed algorithm.
\end{abstract}

Index Terms-Sensor networks, distributed filtering, Markovian switching topology, event-based scheme, exponential boundedness.

\section{INTRODUCTION}

Wireless sensor networks have been well recognized as one of the most distinguished technological advances in the past decades [3], [13]. Small and inexpensive sensors, which are spread spatially over monitored regions, are connected through wireless links to form sensor networks that work as a foundation of various complicated applications such as battlefield surveillance, traffic control, environmental monitoring, machine health monitoring, and so on. A fundamental issue of these applications is the distributed filtering, namely, estimate the state vector of the target plant by exploiting noisy observations taken from a group of sensors. This problem has been widely investigated under various objectives, see, for

This work was supported in part by the Research Fund for the Taishan Scholar Project of Shandong Province of China, the National Natural Science Foundation of China under Grants 61490701, 61525305, 61473163, 61522309, 61733009, the Royal Society of the UK, and the Alexander von Humboldt Foundation of Germany. The work of Xiao He was also partially supported by the Special Fund of Suzhou-Tsinghua Innovation Leading Action under Grant 2016SZ0202. (Corresponding author: Qinyuan Liu.)

Q. Liu is with the Department of Computer Science and Technology, Tongji University, Shanghai 201804, China. Email: liugy@tongji.edu.cn.

Z. Wang is with the Department of Computer Science, Brunel University London, Uxbridge, Middlesex, UB8 3PH, United Kingdom. Email: Zidong. Wang@brunel. ac.uk.

$\mathrm{X}$. He is with the Department of Automation, Beijing National Research Center for Information Science and Technology (BNRist), Tsinghua University, Beijing 100084, China.

D. H. Zhou is with the College of Electrical Engineering and Automation, Shandong University of Science and Technology, Qingdao 266590, China. $\mathrm{He}$ is also with the Department of Automation, Tsinghua University, Beijing 100084, China. example, [5], [12], [14] for optimal filter design and [34], [9] for $H_{\infty}$ performance.

Although the wireless sensor network provides us with unprecedented opportunities, the limitations of sensor devices and wireless channels do introduce new challenges to the distributed applications. For instance, the tendency of miniaturization of modern sensors in general seriously restricts the battery supply and colorred wireless bandwidth constraints do not allow frequent communication. Therefore, resourceefficient transmission schemes have been a focus of research because of their explicit engineering insights in extending the serving hours of sensor networks. Up to now, a number of resource-efficient schemes have been developed in the literature from various perspectives in order to preserve the network resources. Such schemes include, but are not limited to, optimal routing algorithms, sensor/estimator scheduling strategies and event-based transmission approaches [26], [15]. Among others, special attention has been paid to the eventbased communication strategy because of its cost effectiveness. A guiding ideology behind such a strategy is to reduce the communication frequency through introducing an event generator to decide whether the signals shall be sent to the estimator/controller or not. Recently, a rich body of research results has been reported in the literature on the event-based communication problems, see e.g., [15], [8], [20], [6], [29]. To be specific, in [29], the stability has been investigated for event-based networked control systems. By transforming the event-induced error into a system delay model, a sufficient condition that guarantees the stability of closed-loop systems has been obtained by utilizing linear matrix inequalities. In [15], the event-based distributed filtering problems have been considered in order to obtain the optimal estimation. Note that, it is generally impossible to compute the error covariance in a closed form primarily due to the event-induced error, and an alternative way is therefore to design the suboptimal filters by minimizing certain upper bound of error covariance.

It has now been well recognized that the communication networks may suffer from some degree of topological changes due to potentially harsh, uncertain, and dynamic wireless environments, and this gives rise to the so-called dynamic topology problem that have drawn considerable research interest in the context of consensus problems for multi-agent systems, see e.g., [25], [28], [33], [30], [4]. In [28], the stochastic link failure, which stems from the irregular detection areas of agents during the information exchange, has been thoroughly investigated. The dynamically changing interaction topologies have been addressed in [25], where the consensus has been shown to be achieved if the union of the directed 
interaction graphs has a spanning tree. As for the stochastic topologies, it has been proved in [33] that the mean topology determines whether the consensus of single-integrator agent can be achieved, and this result has then been extended to the case of Markovian switching topology in [30]. Although the issue of stochastically switching topologies has been attracting more attention in the community of multi-agent systems, the corresponding results concerning distributed filtering problems are scattered despite the profound application insights of wireless sensor networks.

Recently, some results have been available in the literature on the event-based distributed filtering problems, see e.g. [23], [16]. Nonetheless, under the Markovian switching topologies, the topologies may stochastically change among a set of disconnected graphs at every sampling instant, which substantially impedes the information exchange between sensors and thus hinders the cooperation of the distributed filters. Consequently, in order to guarantee the satisfactory filtering performance (e.g. mean-square boundedness of the estimation errors) for the distributed filtering dynamics, there is an urgent need to develop algorithms capable of accommodating the topology switching while achieving adequate estimation accuracy. As such, the focus of this paper is on the event-based distributed filtering problems for wireless sensor networks over Markovian switching topologies.

The main challenges we are facing can be highlighted as follows: 1) how to define the triggering rule for each state with an individual threshold; 2) what is the influence of the Markovian switching topologies on the distributed filters; and 3) under which conditions the exponential mean-square boundedness of the estimation error dynamics for the distributed filtering system can be guaranteed? These challenges are properly handled in this paper by developing a novel event-based distributed filtering algorithm. Specifically, a set of distributed event schedulers are deployed in every sensor to check its individual state so that the communication is only executed whenever an individual triggering condition is satisfied. By exploiting the stochastic analysis techniques and the graph theory, we conclude that the event-based distributed filters with Markovian switching topologies can achieve exponential mean-square boundedness if the sensor network is distributively detectable and the combined interaction topology is strongly connected. A numerical example is utilized to demonstrate the practical significance of the developed algorithm.

Notations. The notations used in this paper are standard (or otherwise will be clarified as we proceed). $\mathbb{R}^{n}$ denotes the $n$-dimensional Euclidean space, $\mathbb{R}^{n \times m}$ is the set of all $n \times m$ matrices and $\mathbb{R}^{+}$represents the set of all positive real numbers. $\mathbb{E}\{x\}$ denotes the mathematical expectation of a random vector $x, \mathbb{E}\{x \mid y\}$ represents the conditional expectation of $x$ given $y$ and $\mathbb{P}\{\cdot\}$ stands for the occurrence probability of the event ".". We refer to $\operatorname{col}\left\{x_{1}, x_{2}, \cdots, x_{n}\right\}$ as the column vector $\left\{x_{1}^{\prime}, x_{2}^{\prime} \cdots, x_{n}^{\prime}\right\}^{\prime}$ and $\mathbf{0}_{n \times m}$ as a $n \times m$ zero matrix. $\operatorname{diag}\left\{x_{1}, x_{2}, \cdots, x_{n}\right\}$ is a block diagonal matrix with the $i$ th block being $x_{i}$ and all other entries being zero. $\lambda_{\max }(A)\left(\lambda_{\min }(A)\right)$ is the eigenvalue of the matrix $A$ with the largest (smallest) modulus. $\delta(\cdot)$ represents a Dirac delta function with the properties

$$
\delta(t)=\left\{\begin{array}{ll}
0 & \text { for } t \neq 0 \\
\infty & \text { for } t=0
\end{array} \quad \text { and } \quad \int_{-\infty}^{\infty} \delta(t) d t=1 .\right.
$$

\section{PRoblem Formulation}

\section{A. The Markovian switching topology}

Directed graphs can be utilized to describe the communication connections of the individual sensors in sensor networks. A directed graph is denoted by $\mathcal{G}=(\mathcal{V}, \mathcal{E}, \mathcal{A})$ with a vertex set $\mathcal{V}=\left\{v_{1}, v_{2}, \cdots, v_{N}\right\}$, an edge set $\mathcal{E} \subseteq \mathcal{V} \times \mathcal{V}$, and an adjacency matrix $\mathcal{A}=\left[a_{i j}\right]_{N \times N}$ with nonnegative elements $a_{i j}$. The edge set represents the topology connections between nodes. Note that $\left(v_{i}, v_{j}\right) \in \mathcal{E}$, if and only if $a_{i j}>0$, indicating that the $i$ th node can receive the data from the $j$ th node. We do not allow the self-loops, namely, $a_{i i}=0$, $\forall i \in \mathcal{V}$. The set of neighbors of $i$ th node is denoted by $N_{i}=\left\{v_{j} \in \mathcal{V} \mid\left(v_{i}, v_{j}\right) \in \mathcal{E}\right\}$. The Laplacian matrix for $\mathcal{G}$ is defined as $H=\mathcal{D}-\mathcal{A}$, where $\mathcal{D}=\operatorname{diag}\left\{d_{1}, d_{2} \cdots, d_{N}\right\}$ is a diagonal matrix with the entries $d_{i}=\sum_{j=1}^{N} a_{i j}$. The graph is said to be strongly connected if every node is reachable from every other node.

In the paper, we suppose that the sensor nodes are deployed with the communication connections subject to a set of time-varying graphs $\mathcal{G}(r(t))=(\mathcal{V}, \mathcal{E}(r(t)), \mathcal{A}(r(t)))$. The evolution of the graph $\mathcal{G}(r(t))$ is governed by a homogeneous continuous-time Markov process $\{r(t)\}$ (with right continuous trajectories) taking values on a finite set $\mathbb{S}=\{1,2, \cdots, S\}$. As such, we have the corresponding adjacency matrix $\mathcal{A}(r(t))$, the set of neighbors $N_{i}(r(t))$ and the Laplacian matrix $H(r(t))$. The transition probabilities of the Markov process are given by:

$\mathbb{P}\{r(t+\Delta t)=j \mid r(t)=i\}= \begin{cases}\pi_{i j} \Delta t+o(\Delta t), & \text { if } i \neq j \\ 1+\pi_{i i} \Delta t+o(\Delta t), & \text { elsewise }\end{cases}$

where $\left[\pi_{i j}\right]_{S \times S}$ is a finite-dimensional transition rate matrix of the Markov chain with $\pi_{i j} \geq 0$ for $i \neq j$ and $\pi_{i i}=$ $-\sum_{j \neq i} \pi_{i j}$. The term $o(\Delta t)$ represents an infinitesimal of higher order than $\Delta t$, i.e., $\lim _{\Delta t \rightarrow 0} o(\Delta t) / \Delta t=0$.

\section{B. The wireless sensor network}

Consider the target plant described by the following nonlinear continuous-time stochastic system:

$$
\dot{x}(t)=f(x(t), t)+b(t) w(t)
$$

where $x(t)=\left(x_{1}(t), x_{2}(t), \cdots, x_{n}(t)\right)^{\prime} \in \mathbb{R}^{n}$ is the internal state of the plant, $f(x)=\left(f_{1}(x), f_{2}(x), \cdots, f_{n}(x)\right)^{\prime} \in \mathbb{R}^{n}$ is a nonlinear function which contributes to the dynamical evolution of the target plant, $b(t)=\left(b_{1}(t), b_{2}(t), \cdots\right.$, $\left.b_{n}(t)\right)^{\prime} \in \mathbb{R}^{n}$ represents the noise intensity, and $w(t)$ is a scalar Gaussian random variable with zero mean and autocorrelation $\mathbb{E}\{w(t) w(t+\tau)\}=\delta(\tau)$.

For every $i \in \mathcal{V}$, the measurement of sensor $i$ is given as follows:

$$
y_{i}(t)=C_{i} x(t)+v_{i}(t) \zeta_{i}(t),
$$


where $y_{i}(t) \in \mathbb{R}^{m_{i}}$ is the measurement signal, $v_{i}(t)=\left(v_{i 1}(t)\right.$, $\left.v_{i 2}(t), \cdots, v_{i n}(t)\right)^{\prime} \in \mathbb{R}^{n}$ represents the noise intensity, and $\zeta_{i}(t) \in \mathbb{R}$ is a scalar Gaussian random variable with zero mean and autocorrelation $\mathbb{E}\left\{\zeta_{i}(t) \zeta_{j}(t+\tau)\right\}=\delta(i-j) \delta(\tau) . C_{i}$ is the measurement matrix of appropriate dimensions. The random variables $r(t), w(t)$ and $\zeta_{i}(t)$ are mutually independent, and the components of the vectors $b(t)$ and $v_{i}(t)$ are bounded, i.e., $0<b_{k}(t) \leq \bar{b}_{k}$ and $0<v_{i k}(t) \leq \bar{v}_{i k}$, for $i \in \mathcal{V}$ and $k=1,2, \cdots, n$, where $\bar{b}_{k}$ and $\bar{v}_{i k}$ are positive scalars. The system (1)-(2) under consideration is assumed to satisfy the following assumptions.

Assumption 1 ([32]): The nonlinear function $f(\cdot): \mathbb{R}^{n} \rightarrow$ $\mathbb{R}^{n}$ is analytic everywhere and satisfies the following condition

$$
\begin{aligned}
&(x-y)^{\prime} P(f(x, t)-f(y, t)-\Delta(x-y)) \\
& \leq-\eta(x-y)^{\prime}(x-y), \quad \forall x, y \in \mathbb{R}^{n}
\end{aligned}
$$

where $P=\operatorname{diag}\left\{p_{1}, p_{2}, \cdots, p_{n}\right\}, \Delta=\operatorname{diag}\left\{\delta_{1}, \delta_{2}, \cdots, \delta_{n}\right\}$, and $\eta, p_{i}, \delta_{i}$ are positive scalars.

Assumption 2: The continuous-time Markov process $\{r(t)\}$ with the transition rate matrix $\left[\pi_{i j}\right]_{S \times S}$ is ergodic.

Remark 1: Under the ergodic assumption, the state of the Markov process can be reached from any other state and, for any initial distribution, there always exists a unique stationary distribution $\left\{\bar{\pi}_{i}>0, i \in \mathbb{S}\right\}$ with $\sum_{i=1}^{n} \bar{\pi}_{i}=1$. Moreover, when the Markov process is started off initially with such a stationary distribution, the distribution will be invariant over time.

\section{The event-based distributed filter}

A fundamental issue of the filtering problems is to estimate the state vector $x(t)$ based on the noisy measurements. It is noted that, different from the classical single filter case, the target plant is now observed by a group of smart sensors, and the estimation is carried out in every sensor node in a distributive fashion. Due to the physical limitations of the sensors, the individual sensor usually has insufficient abilities to estimate the plant state based on the local measurements only. To achieve high accuracy estimation, we need to utilize the complementary messages gathered from all the local and neighboring nodes. As such, the distributed filtering algorithm is chosen as follows:

$$
\left\{\begin{aligned}
\frac{d \hat{x}_{i}(t)}{d t} & =f\left(\hat{x}_{i}(t), t\right)+c L_{i}\left(y_{i}(t)-C_{i} \hat{x}_{i}(t)\right) \\
& +c \sum_{j \in N_{i}(r(t))} a_{i j}(r(t))\left(\hat{x}_{j}(t)-\hat{x}_{i}(t)\right) \\
\hat{x}_{i}(0) & =0
\end{aligned}\right.
$$

where $\hat{x}_{i}(t) \in \mathbb{R}^{n}$ is the local estimate of the full state vector $x(t)$ from the $i$ th sensor. The filter gain $L_{i} \in \mathbb{R}^{n \times m}$ and the strength $c \in \mathbb{R}$ are parameters to be designed. According to the above structure, every sensor calculates the local estimate based on its measurements $y_{i}(t)$ as well as the estimates $\hat{x}_{i}(t)$ received from the neighboring sensors.

When taking the energy and bandwidth restrictions into account, some new challenges arise for the distributed filtering problem. As shown in (4), in order to compute the local state estimates, one needs to use the real-time neighboring estimates transmitted via wireless networks. Note that the network communication constitutes one of the main sources for the consumption of the constrained resources. Too frequent transmissions will inevitably put more burden on the network and deteriorate the network performance leading to some unfavorable phenomena such as packet losses and communication delays. As such, in this paper, an event-based scheme is introduced to determine whether the local information will be broadcast or not.

To begin with, we define a sequence of event instants for the $s$ th entry of the estimate $\hat{x}_{i}(t)$ (i.e., $\hat{x}_{i, s}(t)$ ) by a monotonically increasing sequence $0=t_{i, s}^{0}<t_{i, s}^{1}<$ $t_{i, s}^{2}<\cdots$ and the broadcast is triggered only at these event instants. Such a sequence is determined iteratively on-line according to the following rule $t_{i, s}^{k+1}=\inf \left\{t \in \mathbb{R}^{+} \mid t>\right.$ $\left.t_{i, s}^{k}, \hbar_{i, s}\left(\hat{x}_{i, s}(t), \hat{x}_{i, s}\left(t_{i, s}^{k}\right), t\right)>0\right\}$, where the event function $\hbar_{i, s}(\cdot): \mathbb{R} \times \mathbb{R} \times \mathbb{R}^{+} \rightarrow \mathbb{R}$ is chosen to be

$$
\hbar_{i, s}\left(\hat{x}_{i, s}(t), \hat{x}_{i, s}\left(t_{i, s}^{k}\right), t\right)=\left(\hat{x}_{i, s}(t)-\hat{x}_{i, s}\left(t_{i, s}^{k}\right)\right)^{2}-\sigma_{i, s}
$$

for $i \in \mathcal{V}$ and $s=1,2, \cdots, n$ with the individual thresholds $\sigma_{i, s}$ being positive scalars. From the above event-based mechanism, it can be seen that, whenever the triggering condition $\hbar_{i, s}\left(\hat{x}_{i, s}(t), \hat{x}_{i, s}\left(t_{i, s}^{k}\right), t\right)>0$ is satisfied, a broadcast of $\hat{x}_{i, s}(t)$ is triggered with the marked event instant $t_{i, s}^{k+1}$ such that $\hat{x}_{i, s}(t)=\hat{x}_{i, s}\left(t_{i, s}^{k+1}\right)$ and thus that $\hbar_{i, s}\left(\hat{x}_{i, s}(t), \hat{x}_{i, s}\left(t_{i, s}^{k+1}\right), t\right)$ is equal to $-\sigma_{i, s}$. As such, the following inequalities are always true

$$
\hbar_{i, s}\left(\hat{x}_{i, s}(t), \hat{x}_{i, s}\left(t_{i, s}^{k}\right), t\right) \leq 0
$$

For the purpose of clarity, we augment all the components at the latest event instants $\hat{x}_{i}^{t}(t) \triangleq \operatorname{col}\left\{\hat{x}_{i, 1}\left(t_{i, 1}^{k_{i, 1}(t)}\right), \cdots, \hat{x}_{i, n}\left(t_{i, n}^{k_{i, n}(t)}\right)\right\} . \quad$ where $k_{i, j}(t)=\max \left\{\kappa \in \mathbb{N} \mid t_{i, j}^{\kappa}<t\right\}$. According to the event-based mechanism, let us revise (4) by only using the neighboring information at their latest event instants. In the event-based case, the distributed filter is given as follows:

$$
\begin{aligned}
\frac{d \hat{x}_{i}(t)}{d t}=f\left(\hat{x}_{i}(t), t\right) & +c L_{i}\left(y_{i}(t)-C_{i} \hat{x}_{i}(t)\right) \\
+ & c \sum_{j \in N_{i}(r(t))} a_{i j}(r(t))\left(\hat{x}_{j}^{t}(t)-\hat{x}_{i}^{t}(t)\right)
\end{aligned}
$$

which is further rewritten as below:

$$
\begin{gathered}
\frac{d \hat{x}_{i}(t)}{d t}=f\left(\hat{x}_{i}(t), t\right)-c \sum_{j=1}^{N} h_{i j}(r(t)) \hat{x}_{j}^{t}(t) \\
+c L_{i}\left(y_{i}(t)-C_{i} \hat{x}_{i}(t)\right),
\end{gathered}
$$

where $h_{i j}(r(t))$ is the $(i, j)$ th element of the Laplacian matrix $H(r(t))$ associated with the graph $\mathcal{G}(r(t))$.

Denote the event-induced and estimation errors by $e_{i}^{t}(t) \triangleq$ $\hat{x}_{i}^{t}(t)-\hat{x}_{i}(t)$ and $e_{i}(t) \triangleq \hat{x}_{i}(t)-x(t)$, respectively. By subtracting (6) from (1), we can obtain the dynamics of the estimation error as follows:

$$
\frac{d e_{i}(t)}{d t}=f\left(\hat{x}_{i}(t), t\right)-f\left(x_{i}(t), t\right)-c L_{i} C_{i} e_{i}(t)
$$




$$
\begin{aligned}
& -c \sum_{j=1}^{N} h_{i j}(r(t)) e_{j}(t)+c L_{i} v_{i}(t) \zeta_{i}(t) \\
& -c \sum_{j=1}^{N} h_{i j}(r(t)) e_{j}^{t}(t)-b(t) w(t) .
\end{aligned}
$$

Before proceeding, we introduce the following definition for the exponential mean-square boundedness.

Definition 1: The dynamics of the estimation error in (7) is said to be exponentially bounded in mean square if there exist real numbers $\rho>0, \tau>0$ and $\mu>0$ such that

$$
\sum_{i=1}^{N} \mathbb{E}\left\{\left\|e_{i}(t)\right\|^{2}\right\} \leq \rho e^{-\tau t} \sum_{i=1}^{N} \mathbb{E}\left\{\left\|e_{i}(0)\right\|^{2}\right\}+\mu .
$$

where $\tau$ is the decay rate and $\mu$ is the ultimate bound.

Remark 2: It is noteworthy that the mean-square boundedness of error dynamics could be regarded as an important criterion judging whether the filtering algorithm is feasible or not, and therefore it is widely considered in many works, see for example [7], [10], [21].

The objective of this paper is to design the parameter $c$ and the filter gains $L_{i}$ for each sensor such that the dynamics of the distributed estimation error (7) is exponentially bounded in the mean-square sense.

\section{MAIN RESULTS}

In this section, a sufficient criterion will be established to guarantee the exponential mean-square boundedness of the estimation errors for the proposed event-based distributed filtering algorithm over Markovian switching topologies.

A useful lemma is presented as follows.

Lemma 1 ([31]): Suppose that a directed graph $\mathcal{G}$ with the Laplacian matrix $H$ is strongly connected. Then, there exists a positive vector $\xi=\left(\xi_{1}, \xi_{2}, \cdots, \xi_{N}\right)^{\prime}$ such that $\xi^{\prime} H=0$. Furthermore, $(H)^{s} \triangleq\left(\Xi H+H^{\prime} \Xi\right)$ is a symmetric matrix with zero row sum, where $\Xi=\operatorname{diag}\left\{\xi_{1}, \xi_{2}, \cdots, \xi_{N}\right\}$.

To continue the discussion, we need to introduce the definition for the distributive detectability as follows,

Definition 2: A sensor network described by (1)-(2) is said to be distributively detectable if there exist matrices $L_{i}$ satisfying

$$
\begin{aligned}
P L_{i} C_{i}+ & C_{i}^{\prime} L_{i}^{\prime} P \geq 2 \sum_{k \in \mathcal{M}_{i}} \Theta_{i k}, \\
\bigcup_{\forall i \in \mathcal{V}} \mathcal{M}_{i} & =\{k\}_{k=1}^{n},
\end{aligned}
$$

where $P$ is a positive definite diagonal matrix defined in Assumption $1, \mathcal{M}_{i}$ is any arbitrary subset of $\{k\}_{k=1}^{n}$, and $\Theta_{i k}$ is a diagonal matrix of the form

$$
\Theta_{i k} \triangleq \operatorname{diag}\{\underbrace{0, \cdots, 0,}_{k-1} \gamma_{i k}, \underbrace{0, \cdots, 0}_{n-k}\},
$$

with a positive real number $\gamma_{i k}>0$.

Remark 3: For linear time-invariant systems, it has been shown that the mean-square boundedness can be achieved when $(A, C)$ is distributively observable, where $C=$ $\left[C_{1} ; C_{2} ; \ldots ; C_{m}\right]$. Unfortunately, as the system in (1) has nonlinear dynamics, the observability of linear time-invariant systems is inapplicable. In this case, we need to establish another type of distributive detectability to guarantee the boundedness of the estimation error dynamics.

The distributive detectability property plays an essential role in guaranteeing the boundedness of the estimation error dynamics. For a distributive detectable system, the following condition always holds:

$$
x^{\prime}(t) P\left(L_{i} C_{i} x(t)\right) \geq \sum_{k \in \mathcal{M}_{i}} \gamma_{i k} x_{k}^{\prime}(t) x_{k}(t),
$$

from which we can see that the $k$ th $\left(k \in \mathcal{M}_{i}\right)$ entry of the state vector can be extracted by sensor $i$. Notice that the union of all the subsets equals to the whole set, i.e., $\bigcup_{\forall i \in \mathcal{V}} \mathcal{M}_{i}=\{k\}_{k=1}^{n}$. In this case, we will show that the sensor network can complete the distributed estimation from a cooperative perspective.

For simplicity, we introduce the notations $e(t) \triangleq\left[e_{1}^{\prime}(t), e_{2}^{\prime}(t), \quad \cdots, e_{N}^{\prime}(t)\right]^{\prime}, \quad e^{t}(t) \triangleq$ $\left[\left(e_{1}^{t}(t)\right)^{\prime},\left(e_{2}^{t}(t)\right)^{\prime}, \cdots,\left(e_{N}^{t}(t)\right)^{\prime}\right]^{\prime}, \quad \tilde{e}_{k}(t) \triangleq$ $\left[e_{1, k}(t), e_{2, k}(t), \cdots, e_{N, k}(t)\right]^{\prime}$ and $\widehat{\Theta}_{i k} \triangleq$ $\operatorname{diag}\{\underbrace{0, \cdots, 0}_{i-1}, \gamma_{i k}, \underbrace{0, \cdots, 0}_{N-i}\}$, where $e_{i, k}(t)$ is the $k$ th element of the vector $e_{i}(t)$. Additionally, we denote the set gathering all the sensors that can extract the $k$ th entry of the target state by $\mathcal{M}_{k}^{-} \triangleq\left\{i: k \in \mathcal{M}_{i}\right.$, for $\left.i \in \mathcal{V}\right\}$.

When the sensor network has the distributive detectability as defined in Definition 2, it can be verified that $\bigcup_{\forall k \in\{1, \cdots, n\}} \mathcal{M}_{k}^{-}=\mathcal{V}$. Furthermore, the ergodicity of the Markov chain ensures that there exists a unique stationary distribution $\left\{\bar{\pi}_{i}>0, i \in \mathbb{S}\right\}$. Without loss of generality, we assume that the Markov process starts from such a stationary distribution. As such, it can be seen that $\mathbb{P}\{r(t)=i\}=$ $\bar{\pi}_{i}>0, \forall t \geq 0$. Moreover, we define the weighted Laplacian matrix as $\widehat{H} \triangleq \sum_{l=1}^{S} \bar{\pi}_{l} H(l)$ whose corresponding graph is called the combined graph. According to Lemma 1, we can compute the matrix $\Xi$ with respect to the weighted matrix $\widehat{H}$ if the combined graph is strongly connected. Let $\xi^{-}=\min \left\{\xi_{1}, \xi_{2}, \cdots, \xi_{n}\right\}, \xi^{+}=\max \left\{\xi_{1}, \xi_{2}, \cdots, \xi_{n}\right\}, p^{-}=$ $\min \left\{p_{1}, p_{2}, \cdots, p_{n}\right\}$, and $p^{+}=\max \left\{p_{1}, p_{2}, \cdots, p_{n}\right\}$.

Now, we are in the position to obtain a sufficient condition for the exponential mean-square boundedness in the following theorem.

Theorem 1: Consider the system (1)-(2) subject to Markovian switching topologies. Under Assumptions 1-2, if the following criteria

$$
\left(2 p_{k} \delta_{k}+1\right) \Xi-c\left(p_{k} \widehat{H}+\sum_{i \in \mathcal{M}_{k}^{-}} \widehat{\Theta}_{i k}\right)^{s}<0
$$

are satisfied for $k=1,2, \cdots, n$, then the filtering error dynamics (7) is exponentially mean-square bounded with the decay rate $\tau<2 \eta / p^{+}$and the ultimate bound $\mu=\tilde{\mu} / \tau \xi^{-} p^{-}$ where

$$
\begin{aligned}
\tilde{\mu}= & \sum_{i=1}^{N} \sum_{k=1}^{n} \xi_{i} p_{k} \bar{b}_{k}^{2}+c^{2} p^{+} \sum_{i=1}^{N} \sum_{k=1}^{n} \xi_{i} \lambda_{\max }\left(L_{i}^{\prime} L_{i}\right) \bar{v}_{i k}^{2} \\
& +c^{2} \xi^{+} \lambda_{\max }\left(\widehat{H}^{\prime} \widehat{H}\right) \sum_{i=1}^{N} \sum_{k=1}^{n} p_{k} \sigma_{i, k} .
\end{aligned}
$$


Proof: Construct the following Lyapunov-like function for system (7):

$$
V(e(t), j)=\sum_{i=1}^{N} \xi_{i} e_{i}^{\prime}(t) P_{j} e_{i}(t) .
$$

where $P_{j}$ are positive definite matrices. Denote the infinitesimal operator $\mathcal{L}(\cdot)$ of the random process $\{e(t), r(t)\}$ by

$$
\begin{aligned}
& \mathcal{L} V(e(t), j) \\
\triangleq & \lim _{\Delta t \rightarrow 0^{+}} \frac{1}{\Delta t}\{\mathbb{E}\{V(e(t+\Delta t), r(t+\Delta t)) \mid r(t)=j, e(t)\} \\
& -V(e(t), j)\},
\end{aligned}
$$

then, we have (see [19], [22])

$$
\begin{aligned}
\mathcal{L} V & (e(t), j) \\
= & \sum_{i=1}^{N}\left\{2 \xi _ { i } e _ { i } ^ { \prime } ( t ) P _ { j } \left(f\left(\hat{x}_{i}(t), t\right)-f\left(x_{i}(t), t\right)\right.\right. \\
& -c \sum_{j=1}^{N} h_{i j}(r(t)) e_{j}(t)-c \sum_{j=1}^{N} h_{i j}(r(t)) e_{j}^{t}(t) \\
& \left.-c L_{i} C_{i} e_{i}(t)\right)+c^{2} \xi_{i} v_{i}^{\prime}(t) L_{i}^{\prime} P_{j} L_{i} v_{i}(t) \\
& \left.+\xi_{i} b^{\prime}(t) P_{j} b(t)\right\}+\sum_{l=1}^{S} \pi_{j l} V(e(t), l) .
\end{aligned}
$$

From Assumption 1, we have

$$
\begin{aligned}
& e_{i}^{\prime}(t) P\left(f\left(\hat{x}_{i}(t), t\right)-f\left(x_{i}(t), t\right)\right) \\
& \quad \leq-\eta e_{i}^{\prime}(t) e_{i}(t)+e_{i}^{\prime}(t) P \Delta e_{i}(t) .
\end{aligned}
$$

Moreover, through some algebraic manipulations, it can be verified that

$$
\begin{aligned}
& 2 \sum_{i=1}^{N} \xi_{i} e_{i}^{\prime}(t) P \Delta e_{i}(t) \\
& =2 \sum_{i=1}^{N} \sum_{k=1}^{n} \xi_{i} e_{i, k}^{\prime}(t) p_{k} \delta_{k} e_{i, k}(t) \\
& =2 \sum_{k=1}^{n} p_{k} \delta_{k} \tilde{e}_{k}^{\prime}(t) \Xi \tilde{e}_{k}(t),
\end{aligned}
$$

and

$$
\begin{aligned}
& 2 \sum_{i=1}^{N} \xi_{i} e_{i}^{\prime}(t) P\left(c \sum_{j=1}^{N} h_{i j}(r(t)) e_{j}(t)\right) \\
& \quad=2 c \sum_{i=1}^{N} \sum_{j=1}^{N} \sum_{k=1}^{n} \xi_{i} e_{i, k}^{\prime}(t) p_{k} h_{i j}(r(t)) e_{j, k}(t) \\
& \quad=c \sum_{k=1}^{n} p_{k} \tilde{e}_{k}^{\prime}(t)(H(r(t)))^{s} \tilde{e}_{k}(t),
\end{aligned}
$$

where $(H(r(t)))^{s}=\Xi H(r(t))+H^{\prime}(r(t)) \Xi$. Furthermore, we have

$$
\begin{aligned}
\sum_{i=1}^{N} \xi_{i} b^{\prime}(t) P b(t) & =\sum_{i=1}^{N} \sum_{k=1}^{n} p_{k} \xi_{i} b_{k}^{\prime}(t) b_{k}(t) \\
& \leq \sum_{i=1}^{N} \sum_{k=1}^{n} p_{k} \xi_{i} \bar{b}_{k}^{2}
\end{aligned}
$$

and

$$
\begin{aligned}
& \sum_{i=1}^{N} c^{2} \xi_{i} v_{i}^{\prime}(t) L_{i}^{\prime} P L_{i} v_{i}(t) \\
& \quad \leq c^{2} p^{+} \sum_{i=1}^{N} \sum_{k=1}^{n} \xi_{i} \lambda_{\max }\left(L_{i}^{\prime} L_{i}\right) v_{i k}^{\prime}(t) v_{i k}(t) \\
& \leq c^{2} p^{+} \sum_{i=1}^{N} \sum_{k=1}^{n} \xi_{i} \lambda_{\max }\left(L_{i}^{\prime} L_{i}\right) \bar{v}_{i k}^{2} .
\end{aligned}
$$

Note that the Markov process $\{r(t)\}$ starts from the invariant distribution $\left\{\bar{\pi}_{i}>0, i \in \mathbb{S}\right\}$. According to [19] and [24], we have

$$
\mathbb{E}\{\mathcal{L} V(e(t), r(t))\}=\sum_{j=1}^{S} \mathbb{E}\left\{\mathcal{L} V(e(t), j) \bar{\pi}_{j}\right\} .
$$

Letting $P_{i}=P$. Substituting (14)-(19) into the above equations and together with $\sum_{j=1}^{S} \pi_{i j}=0$, we obtain

$$
\begin{aligned}
\mathbb{E} & \{\mathcal{L} V(e(t), r(t))\} \\
\leq & \mathbb{E}\left\{-2 \eta \sum_{i=1}^{N} \xi_{i} e_{i}^{\prime}(t) e_{i}(t)\right\}-\mathbb{E}\left\{2 c \sum_{i=1}^{N} \xi_{i} e_{i}^{\prime}(t) P L_{i} C_{i} e_{i}(t)\right\} \\
& +\mathbb{E}\left\{2 \sum_{k=1}^{n} p_{k} \tilde{e}_{k}^{\prime}(t)\left(2 \delta_{k} \Xi-c(\widehat{H})^{s}\right) \tilde{e}_{k}(t)\right\} \\
& +\mathbb{E}\left\{2 \sum_{i=1}^{N} \xi_{i} e_{i}^{\prime}(t) P\left(c \sum_{j=1}^{N} \hat{h}_{i j} e_{j}^{\prime}(t)\right)\right\} \\
& +\sum_{i=1}^{N} \sum_{k=1}^{n} c^{2} p^{+} \xi_{i} \lambda_{\max }\left(L_{i}^{\prime} L_{i}\right) \bar{v}_{i k}^{2}+\sum_{i=1}^{N} \sum_{k=1}^{n} p_{k} \xi_{i} \bar{b}_{k}^{2}
\end{aligned}
$$

where $\hat{h}_{i j}$ is the $(i, j)$ th element of the matrix $\widehat{H}$. Invoking the basic inequality $(x+y)^{\prime}(x+y) \geq 0$, one derives that

$$
\begin{gathered}
-2 \sum_{i=1}^{N} \xi_{i} e_{i}^{\prime}(t) P\left(c \sum_{j=1}^{N} \hat{h}_{i j} e_{j}^{t}(t)\right) \leq \sum_{i=1}^{N} \xi_{i} e_{i}^{\prime}(t) P e_{i}(t) \\
+\sum_{i=1}^{N} \xi_{i}\left(c \sum_{j=1}^{N} \hat{h}_{i j} e_{j}^{t}(t)\right)^{\prime} P\left(c \sum_{j=1}^{N} \hat{h}_{i j} e_{j}^{t}(t)\right) .
\end{gathered}
$$

The second term in the right-hand side of the above inequality can be rewritten as follows:

$$
\begin{aligned}
& \sum_{i=1}^{N} \xi_{i}\left(c \sum_{j=1}^{N} \hat{h}_{i j} e_{j}^{t}(t)\right)^{\prime} P\left(c \sum_{j=1}^{N} \hat{h}_{i j} e_{j}^{t}(t)\right) \\
& =c^{2} e^{t}(t)^{\prime}\left(\left(\widehat{H}^{\prime} \Xi \widehat{H}\right) \otimes P\right) e^{t}(t) \\
& \leq c^{2} \xi^{+} \lambda_{\max }\left(\widehat{H}^{\prime} \widehat{H}\right) \sum_{i=1}^{N} \sum_{k=1}^{n} p_{k} \sigma_{i, k},
\end{aligned}
$$

where the last inequality follows from $\hbar_{i, s}\left(\hat{x}_{i, s}(t), \hat{x}_{i, s}\left(t_{k}^{(i, s)}\right), t\right) \leq 0$. Substituting (21)-(22) into (20) yields that

$$
\begin{aligned}
& \mathbb{E}\{\mathcal{L} V(e(t), r(t))\} \\
& \leq \mathbb{E}\left\{\sum_{k=1}^{n} p_{k} \tilde{e}_{k}^{\prime}(t)\left(2 \delta_{k} \Xi-c(\widehat{H})^{s}+\Xi\right) \tilde{e}_{k}(t)\right\}
\end{aligned}
$$




$$
\begin{aligned}
& -\mathbb{E}\left\{2 c \sum_{i=1}^{N} \xi_{i} e_{i}^{\prime}(t) P L_{i} C_{i} e_{i}(t)\right\}+\tilde{\mu} \\
& -\mathbb{E}\left\{2 \eta \sum_{i=1}^{N} \xi_{i} e_{i}^{\prime}(t) e_{i}(t)\right\},
\end{aligned}
$$

By utilizing the distributed detectability of the sensor network (9), we obtain

$$
\begin{aligned}
& -2 \sum_{i=1}^{N} \xi_{i} e_{i}^{\prime}(t) P\left(c L_{i} C_{i} e_{i}(t)\right) \\
& \leq-2 c \sum_{i=1}^{N} \sum_{k \in \mathcal{M}_{i}} \gamma_{i k} \xi_{i} e_{i, k}^{\prime}(t) e_{i, k}(t) \\
& =-2 c \sum_{k=1}^{n} \sum_{i \in \mathcal{M}_{k}^{-}} \gamma_{i k} \xi_{i} e_{i, k}^{\prime}(t) e_{i, k}(t) \\
& =-2 c \sum_{k=1}^{n} \tilde{e}_{k}^{\prime}(t)\left(\Xi \sum_{i \in \mathcal{M}_{k}^{-}} \widehat{\Theta}_{i k}\right) \tilde{e}_{k}(t) .
\end{aligned}
$$

As such, when the condition in (11) is fulfilled, the following inequality can be derived

$$
\mathbb{E}\{\mathcal{L} V(e(t), r(t))\} \leq-2 \eta \mathbb{E}\left\{\sum_{i=1}^{N} \xi_{i} e_{i}^{\prime}(t) e_{i}(t)\right\}+\tilde{\mu} .
$$

To further prove the exponential mean-square boundedness, we construct a new function as follows:

$$
\mathscr{V}(t, e(t), r(t)) \triangleq e^{\tau t} V(e(t), r(t)),
$$

where $\tau>0$ is a constant to be determined later. It is straightforward to show that

$$
\begin{aligned}
& \mathbb{E}\{\mathcal{L} \mathscr{V}(t, e(t), r(t))\} \\
& \quad=e^{\tau t} \mathbb{E}\{\mathcal{L} V(e(t), r(t))\}+\tau e^{\tau t} \mathbb{E}\{V(e(t), r(t))\} .
\end{aligned}
$$

Note that

$$
\mathbb{E}\{V(e(t), r(t))\} \leq p^{+} \mathbb{E}\left\{\sum_{i=1}^{N} \xi_{i} e_{i}^{\prime}(t) e_{i}(t)\right\} .
$$

Choosing $\tau<2 \eta / p^{+}$, in conjunction with (25), it is not difficult to prove that

$$
\begin{aligned}
& \mathbb{E}\{\mathcal{L} \mathscr{V}(t, e(t), r(t))\} \\
& \leq\left(-2 \eta+\tau p^{+}\right) e^{\tau t} \mathbb{E}\left\{\sum_{i=1}^{N} \xi_{i} e_{i}^{\prime}(t) e_{i}(t)\right\}+e^{\tau t} \tilde{\mu} \leq e^{\tau t} \tilde{\mu} .
\end{aligned}
$$

By using the generalized Itô formula [1], one has

$$
\begin{aligned}
& \mathbb{E}\{\mathscr{V}(t, e(t), r(t))\} \\
& =\mathbb{E}\{\mathscr{V}(0, e(0), r(0))\}+\int_{0}^{t} \mathbb{E}\{\mathcal{L} \mathscr{V}(s, e(s), r(s))\} d s \\
& \leq \mathbb{E}\{V(e(0), r(0))\}+\tilde{\mu} \int_{0}^{t} e^{\tau s} d s .
\end{aligned}
$$

Obviously, we have $\mathbb{E}\{V(e(t), \quad r(t))\}$ $\xi^{-} p^{-} \sum_{i=1}^{N} \mathbb{E}\left\{\left\|e_{i}(t)\right\|^{2}\right\} \quad$ and $\quad \mathbb{E}\{V(e(0), \quad r(0))\} \leq$ $\xi^{+} p^{+} \sum_{i=1}^{N} \mathbb{E}\left\{\left\|e_{i}(0)\right\|^{2}\right\}$. Thus, from (26), it follows that

$$
\sum_{i=1}^{N}\left\|e_{i}(t)\right\|^{2} \leq \frac{\xi^{+} p^{+}}{\xi^{-} p^{-}} e^{-\tau t} \sum_{i=1}^{N}\left\|e_{i}(0)\right\|^{2}+\mu,
$$

which eventually implies that the distributed filtering system is exponentially mean-square bounded. The proof is complete.

The following corollary is readily accessible from Theorem 1.

Corollary 1: Consider the event-based distributed filtering system over Markovian switching topologies. If the sensor network is distributively detectable and the union of the graphs $\left\{\mathcal{G}_{1}, \cdots, \mathcal{G}_{S}\right\}$ is strongly connected, then by choosing the parameter

$$
c>\max _{k} \frac{\left(2 p_{k} \delta_{k}+1\right) \xi^{+}}{\lambda_{\min }\left\{\left(p_{k} \widehat{H}+\sum_{i \in \mathcal{M}_{k}^{-}} \widehat{\Theta}_{i k}\right)^{s}\right\}} .
$$

the filtering error dynamics (7) is exponentially mean-square bounded.

Proof: Because of the distributive detectability of the sensor networks, every component of the state can be observed by at least a sensor node, i.e., $\mathcal{M}_{k}^{-} \neq \emptyset$, for $k=1,2, \cdots, n$, where $\emptyset$ represents an empty set. Since the combined graph is strongly connected, the weighted Laplacian matrix $\widehat{H}$ is irreducible. According to Lemma 1, it is not hard to verify that the matrix $\left(p_{k} \widehat{H}\right)^{s}$ is a new symmetric Laplacian matrix with the eigenvalues satisfying $\lambda_{N}\left(\left(p_{k} \widehat{H}\right)^{s}\right) \geq \cdots, \geq$ $\lambda_{2}\left(\left(p_{k} \widehat{H}\right)^{s}\right)>\lambda_{1}\left(\left(p_{k} \widehat{H}\right)^{s}\right)=0$. As a result, we have $\left(p_{k} \widehat{H}+\sum_{i \in \mathcal{M}_{k}^{-}} \widehat{\Theta}_{i k}\right)^{s}>0$. To this end, it can be seen that, if we choose $c$ according to (27), then the criteria (11) is satisfied and therefore the dynamics of the estimation error is exponentially mean-square bounded, which ends the proof.

Remark 4: In the proposed distributed filter, there are two parameters (i.e., strength $c$ and filter gains $L_{i}$ ) waited to be designed, where $L_{i}$ can be obtained by solving the inequalities in (9) and $c$ can be determined based on (27) in Corollary 1. That is to say, if the conditions in Corollary 1 are satisfied, then by choosing the parameter $c>\max _{k}\left(2 p_{k} \delta_{k}+1\right) \xi^{+} / \lambda_{\min }\left\{\left(p_{k} \widehat{H}+\sum_{i \in \mathcal{M}_{k}^{-}} \widehat{\Theta}_{i k}\right)^{s}\right\}$, the filtering error dynamics must be exponentially meansquare bounded.

Remark 5: Note that the threshold $\sigma_{i, s}$ determines the size of an event domain. A large $\sigma_{i, s}$ will result in the increase of ultimate bound $\tilde{\mu}$ (see (12)) while usually reduce the communication frequency. Particularly, when the thresholds $\sigma_{i, s}=0$, the event-based transmission strategy reduces to the classical clock-driven one.

Remark 6: Although the model under investigation is not general for all the real plants, it can describe a lot of practical systems such as three-tank systems [36] and wind turbine systems [17]. The goal of this paper is to establish an explicit condition guaranteeing the mean-square boundedness of the estimation error. Unfortunately, the nonlinear output would significantly complicate the analysis process making it very difficult to obtain an explicit condition. Therefore, the model 


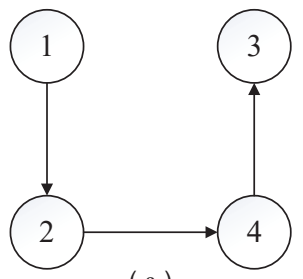

( a )

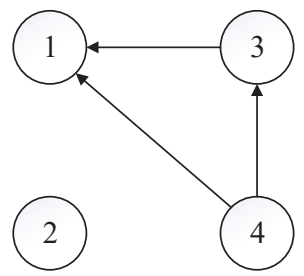

( b )

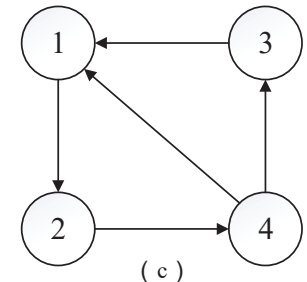

( c )

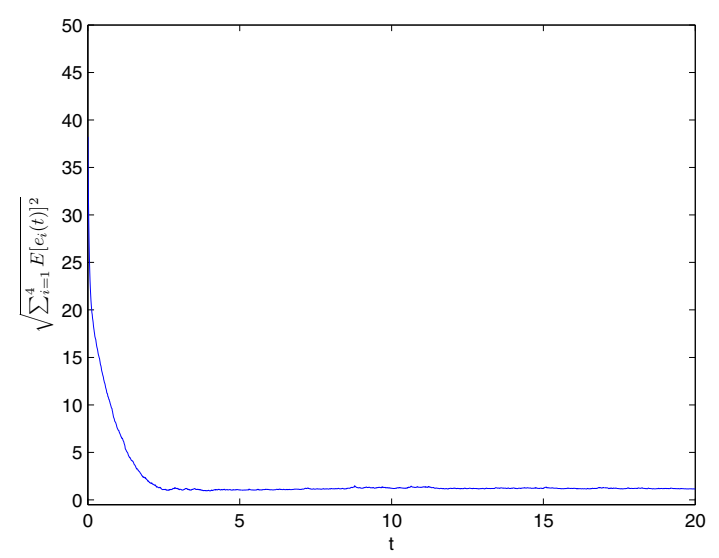

Figure 2. The evolution of the mean-square error

Figure 1. (a) and (b) are the topology of $\mathcal{G}_{1}$ and $\mathcal{G}_{2}$, respectively. (c) is the topology of the combined graph. The combined graph is strongly connected, while neither the separated topology is.

with nonlinear output is beyond the scope of current paper, but it represents a potential topic for our future research.

Remark 7: For estimator design problems of stochastic nonlinear systems subject to Brownian perturbations, an excellent work [18] has been carried out which provides a rigorous mathematical proof of the finite-time input-to-stabilization of the estimation error dynamics. Different from [18], we consider the distributed state estimation in this paper where the nonlinear stochastic system under consideration is subject to both Brownian perturbations and Markovian switching topologies.

\section{Numerical Example}

In this section, a numerical example is provided to demonstrate the applicability of the proposed filtering technique.

The sensor network under consideration is composed of $N=4$ nodes. The directed graphs $\mathcal{G}(r(t))=$ $(\mathcal{V}, \mathcal{E}(r(t)), \mathcal{A}(r(t)))$ with $r(t) \in\{1,2\}$ are depicted in Fig. 1. The adjacency matrices $\mathcal{A}_{1}$ and $\mathcal{A}_{2}$ are given by

$$
\mathcal{A}_{1}=\left[\begin{array}{llll}
0 & 0 & 0 & 0 \\
1 & 0 & 0 & 0 \\
0 & 0 & 0 & 1 \\
0 & 1 & 0 & 0
\end{array}\right], \quad \mathcal{A}_{2}=\left[\begin{array}{llll}
0 & 0 & 1 & 1 \\
0 & 0 & 0 & 0 \\
0 & 0 & 0 & 1 \\
0 & 0 & 0 & 0
\end{array}\right] .
$$

The transmission probability matrix is chosen to be

$$
\left[\pi_{i j}\right]_{2 \times 2}=\left[\begin{array}{cc}
-1 & 1 \\
1 & -1
\end{array}\right] .
$$

Suppose that the initial distribution of the Markov process obeys an invariant distribution $\bar{\pi}_{1}=0.5$ and $\bar{\pi}_{2}=0.5$. The dynamics of the target system is

$$
\left\{\begin{array}{l}
\dot{s}=-s+0.2132 \alpha+0.1521 s^{2} \alpha^{2}+b_{1} w \\
\dot{\alpha}=-0.5000 \alpha-0.1018 \alpha^{3}+b_{2} w
\end{array}\right.
$$

where $s$ represents the position and $\alpha$ is the velocity with the respective initial values -3.70 and 5.50 . It is not hard to verify that the above dynamics satisfies the constraint in Assumption 1 with $P=\operatorname{diag}\{1,1\}, \Delta=\operatorname{diag}\{8,7\}$, and $\eta=0.01$. The measurement matrices are chosen to be

$C_{1}(k)=\left[\begin{array}{ll}10 & 0\end{array}\right], C_{2}(k)=\left[\begin{array}{ll}0 & 2\end{array}\right], C_{3}(k)=\left[\begin{array}{ll}5 & 0\end{array}\right], C_{4}(k)=\left[\begin{array}{ll}0 & 1\end{array}\right]$.

Other parameters are set to be $\sigma_{i k}=0.2, \bar{b}_{k}=1$, and $\bar{v}_{i k}=1$, for $k=1,2$ and $i=1,2,3,4$. Furthermore, the filter gains $L_{i}$ are designed as follows

$$
L_{1}(k)=\left[\begin{array}{ll}
1 & 0
\end{array}\right], L_{2}(k)=\left[\begin{array}{ll}
0 & 3
\end{array}\right], L_{3}(k)=\left[\begin{array}{ll}
2 & 0
\end{array}\right], L_{4}(k)=\left[\begin{array}{ll}
10 & 0
\end{array}\right] .
$$

such that the sensor network is distributively detectable. According Lemma 1, one can obtain

$$
\Xi=\operatorname{diag}\{0.3288,0.6576,0.1644,0.6576\}
$$

From the results of Corollary 1, it is not hard to verify that the criteria (11) can be fulfilled by choosing the strength $c=2$. For the sake of simulating the proposed event-based distributed filtering algorithm, we set the simulation step $d t$ to be $0.01 \mathrm{~s}$ and examine the triggering conditions at each step.

The simulation result is presented in Fig. 2, which depicts the trajectories of the mean-square error of the distributed filters under 1000 independent experiments. The result shows that the estimation error of the distributed filters interacting over Markovian switching topologies is exponentially meansquare bounded. Additionally, the number of events among 2000 steps is presented in Table I, from which we confirm that the communication frequency has been greatly reduced.

Table I

TRIGGERING TIMES IN TOTAL 2, 000 STEPS

\begin{tabular}{ccccc}
\hline Sensor & 1th & 2th & 3th & 4th \\
\hline First element & 321 & 375 & 747 & 413 \\
Second element & 1130 & 705 & 1084 & 1286 \\
\hline
\end{tabular}




\section{CONCLUSION}

This paper has addressed the event-based distributed filtering problems over Markovian switching networks. To focus on specific components of the target state, we have introduced individual triggering conditions by utilizing individual thresholds for different components. We have shown that the exponential mean-square boundedness of distributed estimation error is always achieved if the sensor network is distributively detectable as a whole and the combined communication topology is strongly connected. Finally, the validity of the proposed filtering strategy has been illustrated via numerical simulation. We note that the proposed distributed filter is only valid when the nonlinear function $f(x(t), t)$ satisfies Assumption 1 and the measurement output $y(t)$ is linear. Then, a relevant research topic is to investigate the distributed filter design problem for general nonlinear systems based on the Takagi-Sugeno fuzzy model as [17]. Moreover, another future research topic would be the extension of our main results to more complex situations such as considering time delay or quantization effect in the communication process [27], [2], [35].

\section{REFERENCES}

[1] T. Björk, Finite dimensional optimal filters for a class of Itô processes with jumping parameters, Stochastics-an International Journal of Probability \& Stochastic Processes, vol. 4, no. 2, pp. 167-183, 1980.

[2] R. Caballero-Aguila, A. Hermoso-Carazo, and J. Linares-Perez, Optimal state estimation for networked systems with random parameter matrices, correlated noises and delayed measurements, International Journal of General Systems, vol. 44, no. 2, pp. 142-154, 2015.

[3] C. Chong and S. Kumar, Sensor networks: evolution, opportunities, and challenges, Proceedings of the IEEE, vol. 91, no. 8, pp. 1247-1256, 2003.

[4] C. Ma, T. Li, and J. Zhang, Consensus control for leader-following multiagent systems with measurement noises, Journal of Systems Science and Complexity, vol. 23, no. 1, pp. 35-49, 2010.

[5] F. Cattivelli and A. Sayed, Diffusion strategies for distributed Kalman filtering and smoothing, IEEE Trans. on Automatic Control, vol. 55, no. 9, pp. 2069-2084, 2010.

[6] F. Forni, S. Galeani, D. Nešic, and L. Zaccarian. Event-triggered transmission for linear control over communication channels, Automatica, vol. 50, no. 2, pp. 490-498, 2014.

[7] G. Battistelli and L. Chisci, KullbackCLeibler average, consensus on probability densities, and distributed state estimation with guaranteed stability, Automatica, vol. 50, no. 3, 707-718, 2014.

[8] W. Heemels, M. Donkers, and A. Teel, Periodic event-triggered control for linear systems, IEEE Trans. Automatic Control, vol. 58, no. 4, pp. 847-861, 2012.

[9] C. Huang, D. W. C. Ho, and J. Lu, Partial-information-based distributed filtering in two-targets tracking sensor networks, IEEE Transactions on Circuits and Systems I - Regular Papers, vol. 59, no. 4, pp. 820-832, 2012.

[10] K. Reif, S. Gunther, E. Yaz, and R, Unbehauen, Stochastic stability of the discrete-time extended Kalman filter, IEEE Trans. on Automatic control, vol. 44, no. 4, pp. 714-728, 1999.

[11] B. Kim and R. Baldick, A comparison of distributed optimal power flow algorithms, IEEE Trans. Power Systems, vol. 15, no. 2, pp. 599-604, 2000.

[12] W. Li, Y. Jia, and J. Du, Distributed Kalman consensus filter with intermittent observations, Journal of the Franklin Institute, vol. 352, no. 9, pp. 3764-3781, 2015.

[13] Q. Liu, Z. Wang, X. He, and D. H. Zhou, On Kalman-consensus filtering with random link failures over sensor networks, IEEE Trans. Automatic Control, DOI: 10.1109/TAC.2017.2774601, 2017.

[14] Q. Liu, Z. Wang, X. He, G. Ghinea, and F. E. Alsaadi, A resilient approach to distributed filter design for time-varying systems under stochastic nonlinearities and sensor degradation, IEEE Trans. on Signal Processing, vol. 65, no. 5, pp. 1300-1309, 2017.
[15] Q. Liu, Z. Wang, X. He, and D. Zhou, Event-Based recursive distributed filtering over wireless sensor networks, IEEE Trans. Automatic Control, vol. 60 , no. 9 , pp. $2470-2475,2015$.

[16] Q. Liu, Z. Wang, X. He, and D. Zhou, Event-based filtering with individual triggering thresholds in wireless sensor network: Distributed detectability analysis, IEEE Proc. 34th Chinese Control Conference, Hangzhou, P. R. China, pp. 1734-1739, 2015.

[17] X. Liu, Z. Gao, and Z. Q. Chen, Takagi-Sugeno fuzzy model based fault estimation and signal compensation with application to wind turbines, IEEE Trans. on Industrial Electronics, vol. 64, no. 7, pp. 5678-5689, 2017.

[18] X. Liu and Z. Gao, Robust finite-time fault estimation for stochastic nonlinear systems with Brownian motions, Journal of the Franklin Institute, vol. 354, no. 6, pp. 2500-2523, 2017.

[19] W. Li and Z. Wu, Output tracking of stochastic high-order nonlinear systems with Markovian switching, IEEE Trans. Automatic Control, vol. 58 , no. 6, pp. 1585-1590, 2013.

[20] M. Lemmon, Event-triggered feedback in control, estimation, \& optimization. In A. Bemporad, M. Heemels and M. Johansson (Eds.), Networked control systems, London: Springer, pp. 293-358, 2010.

[21] Y. Li, Y. Sun and F. Meng, New criteria for exponential stability of switched time varying systems with delays and nonlinear disturbances, Nonlinear Analysis:Hybrid Systems, vol. 26, pp. 284-291, 2017.

[22] X. Mao, Exponential stability of stochastic delay interval systems with Markovian switching, IEEE Trans. Autom. Control, vol. 47, no. 10, pp. 1604-1612, Oct. 2002.

[23] X. Meng and T. Chen, Optimality and stability of event triggered consensus state estimation for wireless sensor networks, IEEE Proc. 2014 American Control Conference, Portland, Oregon, USA, pp. 35653570, 2014.

[24] S. M. Ross, Stochastic Processes, New York: Wiley, 1996.

[25] W. Ren and R. Beard, Consensus seeking in multi-agent systems under dynamically changing interaction topologies, IEEE Trans. Automatic Control, vol. 50, no. 5, pp. 655-661, 2005.

[26] C. Schurgers and M. Srivastava, Energy efficient routing in wireless sensor networks, MILCOM Proc. Communication for Network-Centric Ops.: Creating the Info. Force, McLean, VA, 2001.

[27] J. Song, Y. Niu, and Y. Zou, Asynchronous sliding mode control of Markovian jump systems with time-varying delays and partly accessible mode detection probabilities, Automatica, vol. 93, pp. 33-41, 2018.

[28] F. Xiao, L. Wang, and T. Chen, Connectivity preservation for multi-agent rendezvous with link failure, Automatica, vol. 48, no. 1, pp. 25-35, 2012.

[29] D. Yue, E. Tian, and Q. Han, A delay system method for designing event-triggered controllers of networked control systems, IEEE Trans. Automatic Control, vol. 58, no. 2, pp. 475-481, 2013.

[30] K. You, Z. Li, and L. Xie, Consensus condition for linear multiagent systems over randomly switching topologies, Automatica, vol. 49, no. 10 , pp. 3125-3132, 2013.

[31] W. Yu, G. Chen, M. Cao, and J. Kurths, Second-order consensus for multi-agent systems with directed topologies and nonlinear dynamics. IEEE Transactions on Systems, Man, and Cybernetics-Part B, vol. 40, no. 3, pp. 881-891, 2010.

[32] W. Yu, G. Chen, and M. Cao, Consensus in directed networks of agents with nonlinear dynamics, IEEE Transactions on Automatic Control, vol. 56, no. 6, pp. 1436-1441, 2011.

[33] Y. Zhang and Y. Tian, Maximum allowable loss probability for consensus of multi-agent systems over random weighted lossy networks, IEEE Trans. Automatic Control, vol. 57, no. 8, pp. 2127-2132, 2012.

[34] S. Zhang, Z. Wang, D. Ding, and H. Shu, $H_{\infty}$ fuzzy control with randomly occurring infinite distributed delays and channel fadings, IEEE Trans. on Fuzzy Systems, vol. 22, no. 1, pp. 189-200, 2014.

[35] M. Zhong, D. Zhou, and S. X. Ding, On designing $H_{\infty}$ fault detection filter for linear discrete time-varying systems, IEEE Transactions on Automatic Control, vol. 55, no. 7, pp. 1689-1695, 2010.

[36] D. H. Zhou, X. He, Z. Wang, G. Liu and Y. Ji, Leakage fault diagnosis for an internet-based three-tank system: an experimental study, IEEE Transactions on Control Systems Technology, vol. 20, no. 4, pp. 857870, 2012. 